\title{
Review of Optical Fiber Sensor Network Technology Based on White Light Interferometry
}

\author{
Wenchao $\mathrm{LI}^{1}$, Yonggui YUAN ${ }^{1}$, Jun YANG ${ }^{1}$, and Libo YUAN ${ }^{2 *}$ \\ ${ }^{1}$ The Key Lab of In-Fiber Integrated Optics, Ministry Education of China, Harbin Engineering University, Harbin \\ 150001, China \\ ${ }^{2}$ Photonics Research Center, School of Electronic Engineering and Automation, Guilin University of Electronics \\ Technology, Guilin 541004, China \\ ${ }^{*}$ Corresponding author: Libo YUAN_Ｅ-mail: lbyuan@vip.sina.com
}

\begin{abstract}
Optical fiber sensor networks (OFSNs) provide powerful tools for large-scale buildings or long-distance sensing, and they can realize distributed or quasi-distributed measurement of temperature, strain, and other physical quantities. This article provides some optical fiber sensor network technologies based on the white light interference technology. We discuss the key issues in the fiber white light interference network, including the topology structure of white light interferometric fiber sensor network, the node connection components, and evaluation of the maximum number of sensors in the network. A final comment about further development prospects of fiber sensor network is presented.
\end{abstract}

Keywords: Optical fiber sensor; white light interference; optical fiber sensor network (OFSN); multiplexed sensing system

Citation: Wenchao LI, Yonggui YUAN, Jun YANG, and Libo YUAN, "Review of Optical Fiber Sensor Network Technology Based on White Light Interferometry," Photonic Sensors, 2021, 11(1): 31-44.

\section{Introduction}

There is a great demand for sensors in large-scale construction structures. Sensor networks can greatly improve people's ability to obtain information and improve efficiency [1]. In the process of traditional electrical sensor networking, it is difficult to avoid the problems of complex wiring, low spatial resolution, high power consumption, and low efficiency. Optical fiber sensor network (OFSN) is composed of optical fiber or optical cable as the sensing medium. It has the characteristics of simple wiring, low power consumption, and high efficiency, which is very suitable for working in harsh environment such as high temperature and high pressure [2]. In addition, the optical fiber sensor is very easy to realize the multiplexing technology, which can reduce the cost of single point sensor, greatly improve the cost performance of the sensing system, and make optical fiber sensor have more advantages than the traditional sensor [3]. The multiplexing technology uses the same interrogation system to query the measurement information of multiple sensors, which not only greatly simplifies the complexity of the system, but also ensures the measurement accuracy and reliability of the system [4]. The multiplexing technologies that have been developed are mainly time division multiplexing

Received: 26 November 2020 / Revised: 15 December 2020

(C) The Author(s) 2021. This article is published with open access at Springerlink.com

DOI: $10.1007 / \mathrm{s} 13320-021-0613-\mathrm{x}$

Article type: Review 
(TDM), frequency division multiplexing (FDM), wavelength division multiplexing (WDM), code division multiplexing (CDM), and space division multiplexing (SDM) [5-8].

In recent years, the research directions of the optical fiber sensor network focus on large capacity, long distance, and high resolution [9]. Therefore, the mixed application research of the aforementioned multiple multiplexing technologies has been extensive studied. The sensor network composed of fiber Bragg gratings (FBGs) mainly adopts the WDM technology. However, due to the limitation of the light source bandwidth, the number of sensors in the FBGs sensor network is still very small. Childers et al. [10] used the frequency domain interrogation technology to demodulate the fiber grating sensor network and realized the reading of 800 FBG sensors in a single fiber. The TDM technology has also been applied to FBG networks, mixing the TDM and WDM technology to increase the capacity of the FBG sensor network [5, 11]. Rao et al. [12] has realized the high-resolution and high-speed wavelength shift detection efficient two-dimensional quasi-distributed OFSN through the mixed application of SDM, TDM, and WDM technologies. Yang et al. [13] used the phase mask technology to fabricate ultra-weak draw tower gratings, and the fabricated large-capacity OFSN could interrogate up to 6000 ultra-weak fiber gratings by TDM + WDM.

One of the advantages of the optical fiber white light interferometer is that multiplexing can be realized very easily. At present, SDM, TDM, and FDM technologies have been widely used in the field of the white light interference sensing technology [14-18]. The optical fiber sensing technology based on white light interferometry is a representative branch in the cross application of the optical fiber sensing technology. It is well known that the coherence length of light is inversely proportional to the bandwidth of the light source. The laser interference technology has a long interference distance, so it can only be used to measure the relative value of the optical path. White light interference sensing technology uses a broadband light source. Due to the wide bandwidth of the light source, its coherence length is very small (about tens of microns). Therefore, the white light interference sensing technology can obtain the absolute value of measured physical quantities. Moreover, the measurement accuracy based on the white light interference sensing technology is closely related to the bandwidth of the light source. Generally, the wider the bandwidth of the light source used in the white light interference sensing system means the higher the measurement accuracy. One of the outstanding advantages of the optical fiber white light interference sensing technology is that it is easy to realize multiplexing. Due to the coherence length of white light interference is very short, it can be realized that multiple sensors do not interfere with each other in their respective coherent length by the simple design. It also has some other advantages. Firstly, it has great advantages in the multiplexing of sensors, which can measure the absolute length and time delay [14]. Secondly, because the coherence length of the sensor signal is short, it can eliminate the time-varying interference of system stray light [16]. Finally, multiple sensing signals can be coherently multiplexed into one signal without the complicated time division multiplexing or frequency division multiplexing technology $[17,18]$.

This paper reviews fiber sensor network technologies that have been developed based on white light interference and their working principles, as well as some of their applications. This paper is arranged as following: in Section 1, the research status of the optical fiber sensor network and the structure of this paper are introduced. In the following section, we review several topological structures of the optical fiber white light interferometric sensor network. In this part, we discuss several typical optical fiber sensor network topologies based on the white light interference 
technology. In Section 3, the key problems in the optical fiber white light interferometric network are discussed, including the node connection devices and the evaluation of the maximum number of sensors in the network. We review some specific application schemes in the fiber optic white light interferometric sensor network and make some related discussion in Section 4. And the last section presents the overall summary and conclusions of this paper, as well as the prospect of future research direction of the optical fiber white light interferometric sensor network technology.

\section{Topological structure of OFSN based on white light interferometry}

The optical fiber white light interferometric sensor network is the same as other optical fiber sensor networks. They are all based on optical fiber which can play the role of signal sensing and signal transmission at the same time. For the multiplexing requirements of a large number of sensors, this dual role of optical fiber must be realized through an appropriate network structure. The topological structure of the OFSN depends on the monitoring structure requirements or the needs of the sensing object, and the connections of the OFSN are realized through fiber fusion splicing, connectors, couplers, and other components. Different from the communication network, the OFSN usually constructs a passive optical fiber sensing local area network around a unique sensing interrogation system. Several typical structures of the OFSN are linear array topology, ring topology, and linear array and ring hybrid ladder topology.

\subsection{Topological structure of linear array optical fiber sensor network}

Figure 1(a) shows a basic linear array sensor network composed of sensing fibers (sensors) connected together by the optical fiber connector. The end of the sensor array is connected to the white light interferometric interrogation system (WLIDS), and the sensor signals are demodulated through the interrogation system. The sensor network connection can also be realized through the $1 \times N$ fiber coupler as shown in Figs.1(b), 1(c), and 1(d). Figure 1(b) constitutes the bus-type optical fiber sensor network topology. In Figs. 1(c) and 1(d), we use $1 \times N$ star couplers to construct a single star OFSN and a composite star OFSN topology. Linear array OFSN is the most common and practical topology of sensor networks.

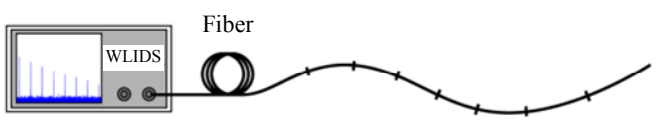

(a)

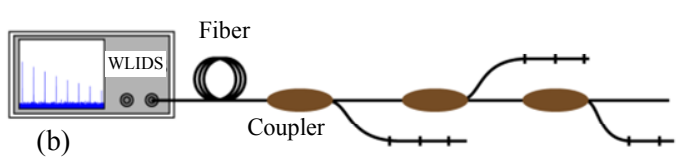

(b)

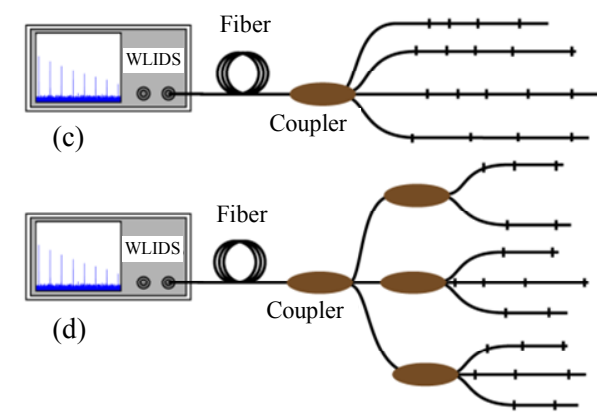

Fig. 1 Topology of the OFSN based on linear array evolution: (a) topological structure of the most basic linear array, (b) bus type, (c) single star, and (d) composite star.

\subsection{Topological structure of the ring optical fiber sensor network}

Aforementioned basic OFSNs are constructed based on reflective optical fiber sensor signals, which just meet the needs of optical fiber white light interferometric sensor systems for reflected interference signals. In fact, for white light interferometric fiber optic sensing systems, a dual-port interrogation system can also be constructed. Due to the dual port interrogation technology, the ring OFSN can effectively avoid the overall fault of the sensor network system. The OFSNs structure based on the double-ended inquiry of the ring topology is shown in Fig. 2, where Fig. 2(a) is the simplest single ring topology. A ring topological structure based on the Sagnac ring is 
shown in Fig.2(b). On the basis of a single ring, a dual-ring OFSN topology evolved through an optical fiber coupler is shown in Figs.2(c) and 2(d).
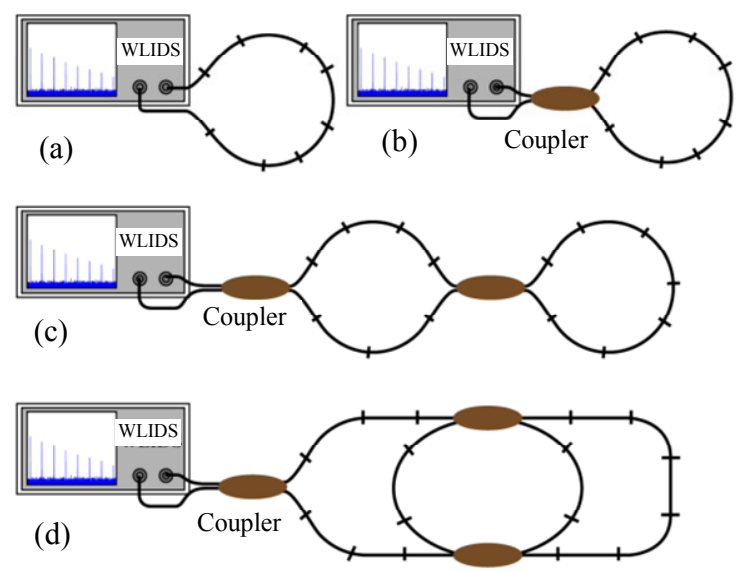

Fig. 2 OFSN topology based on ring sensor array evolution: (a) simplest single ring topology, (b) ring topological structure based on the Sagnac ring, (c) ring topological structure of multi Sagnac ring series connected, and (d) topology of multi Sagnac ring parallel connected.

\subsection{Ladder OFSN topology structure composed by linear array and ring hybrid}

If the aforementioned linear array, the star network, and ring network are topologically combined, a variety of composite OFSN structures can also be evolved. Figure 3(a) shows a hybrid double-ended sensor network topology composed of star array, linear array, and ring network. And a ladder topology based on the hybrid of linear array and ring network is shown in Fig. 3(b). The ladder-type OFSN has the characteristic of both linear array OFSN and ring OFSN, but the topological structure is slightly complicated.

Actually, in the process of constructing an OFSN, not only its topology must be considered, but also various other factors must be considered according to the actual situation, such as multiplexing scheme, number of sensors, power of selected light source, life and reliability of components, and system cost. Obviously, most of these factors are mutually restrictive, and we need to pay attention to the choice of the network structure.
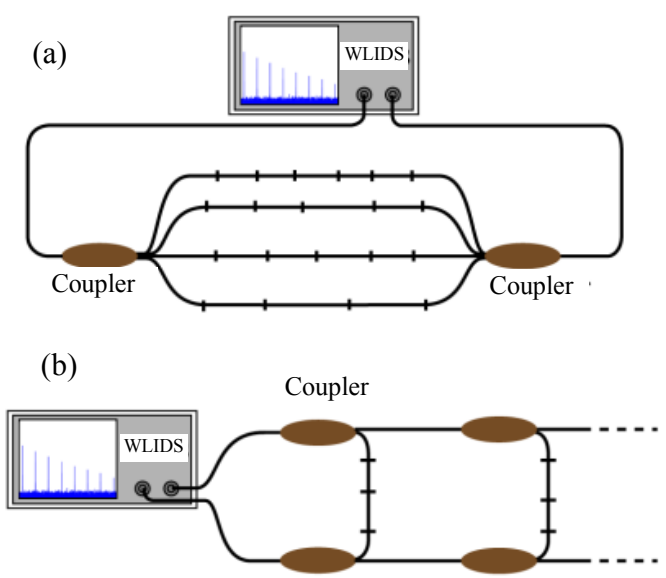

Fig. 3 OFSN structure based on linear and ring hybrid topology evolution: (a) hybrid double-ended sensor network topology based on the star array, linear array, and ring network and (b) ladder topology based on the linear array and ring hybrid.

\section{Key problems of OFSN based on the white light interference technology}

\subsection{Connection devices for network node}

Network node is a key part of the optical fiber sensor network. There are many kinds of devices that can be used as nodes in OFSNs. The network nodes in the fiber white light interferometric sensor network system mainly include fiber couplers, fiber switches, and fiber circulators. In the construction process of the OFSN, these devices play the roles of optical path linking, optical power distribution and energy flow direction control, optical signal splitting and combining, signal selection, and cross interconnection and switching between different networks.

$1 \times 2$ fiber splitter and $2 \times 2$ fiber coupler are the most widely used as network node connection devices in the OFSN. And $1 \times N$ fiber coupler is an indispensable and important component in the construction of a star-type fiber sensor network. The main functions of the fiber coupler in the OFSN are as following: (1) energy flow distribution and direction control; (2) signal separation and combination at the network node; (3) intersection and interconnection of branch optical paths.

Whether in optical communication networks or 
in optical fiber sensor networks, optical switches play the very important role. However, the role of optical switches in OFSNs is different from that of optical fiber communication networks. In optical fiber communication networks, the role of optical switches can greatly reduce the number of connections between communications. In the OFSN, multiple networks of optical fiber sensors share a set of light source system or signal interrogation system through the use of optical switches. In the white light interferometric OFSN system, the optical switch has two main functions: (1) it is used to realize the interconnection between the local area networks formed by multiple passive optical fiber sensors; (2) it is used to share a set of interrogation system to realize selective inquiry of multiple white light interferometric fiber sensor network systems.

The fiber optic circulator is a passive connecting device that controls the direction of beam propagation. Its function is to make the optical signal transmit only in the forward direction and prevent the reverse transmission. Three-port and four-port circulators are the most common fiber circulators. Because the fiber circulator can only transmit in one direction, the main roles of this optical signal transmission direction controller in the OFSN system are: (1) it can be used as a connection device between the light source and sensor network system, and can simultaneously act as an optical isolator; (2) a connection device for sequential transmission of single signals between fiber sensors; (3) a unidirectional interconnection device used between photodetectors (PDs).

\subsection{Evaluation of the maximum number of sensors in a fiber sensor network}

In order to achieve as many sensors as possible in the fiber optic sensor network, the main issue that needs to be considered is power consumption. It mainly includes the following three main factors. The first one is the loss of passive devices in the network. The second one is the power requirement of the fiber sensor itself. And the last one is the minimum signal detection capability of PDs.

(1) Power consumption of passive components in the network: in the OFSN, optical power injected into an optical fiber is distributed to the whole network through several connectors, Y-type or $\mathrm{X}$-type fiber couplers, optical switches, and fiber circulators. Every component in the system absorbs or scatters a certain amount of light power. Usually, the loss of fiber is generally very small, which can be ignored. However, when light needs to transmit a long distance, the optical fiber itself needs to be considered as an optical power consumption component.

(2) Power requirement of sensor itself: on the one hand, optical fiber sensor itself is usually a power consuming element. On the other hand, in order to obtain high-quality signals within a certain dynamic measurement range, there must be certain requirements for optical power. This is usually related to the specific form of the fiber sensor and the dynamic range it measures.

(3) The minimum signal detection capability of the photodetector: the photodetector has a minimum requirement for the minimum signal optical power from the fiber sensor. Usually, the sensor signal is required to be greater than the minimum signal threshold to ensure that the signal is accurately acquired.

A white light interferometric fiber sensor network of the linear array shall be taken as an example to discuss the evaluation method of sensor capacity in the OFSN. A schematic diagram of the white light interferometric linear sensor array based on an adjustable Fabry-Perot (F-P) scanning cavity is shown in Fig. 4. A tunable fiber delay line composed of a scanning prism and two gradient refractive index (GRIN) lenses is inserted into the F-P cavity to match the length of different fiber sensors. The broad-spectrum light emitted by the super luminescent diode (SLD) is coupled into an optical fiber sensor array through the adjustable F-P 
scanning cavity. The sensor array is composed of $N$ segments of sensing fibers ( $N$ sensors) connected end to end. And the connecting surfaces of two adjacent segments of fibers form a series of partial reflectors. The reflected signal returns to the PD along the same optical path.

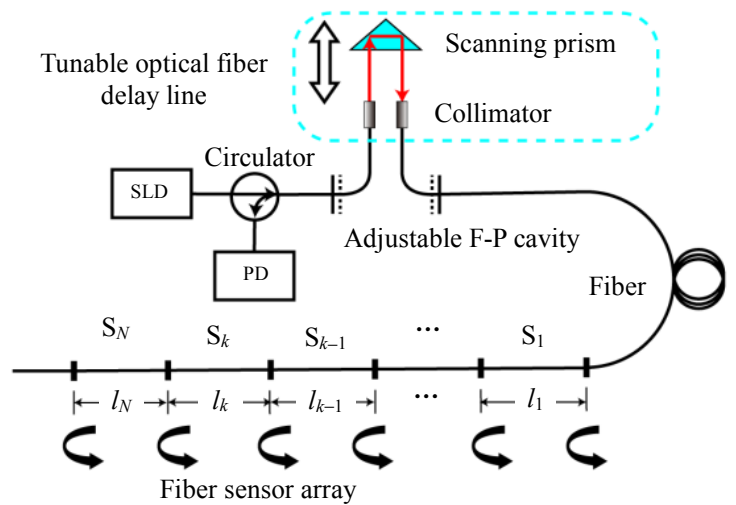

Fig. 4 Working principle of the fiber optic white light interference sensor array based on the adjustable Fizeau scanning cavity.

In the sensor array, the reflectivity of the reflective surface between the sensors is very small ( $1 \%$ or less), which can prevent the input optical signal from attenuating too fast. It should be noted that the length $l_{j}(j=1,2,3, \cdots, N)$ of the fiber optic sensor between two adjacent reflecting surfaces should be made approximately equal but slightly different, so as to distinguish between different sensors. The total optical path of the adjustable F-P scanning cavity is $n L+X$, where $n$ is refractive index of the fiber core, $L$ is the fixed length of the fiber in the adjustable F-P cavity, and $X$ is the adjustable distance of the scanning prism.

When the optical fiber delay line is adjusted to a certain position, the total optical path of the F-P scanning cavity matches the optical path of a certain sensor. At this point, a white light interference fringe will be generated at the output end. The interference fringes come from the reflected signals from the front and back of the sensor, corresponding to the unique sensor.

Taking the $j$ th sensor as an example, its optical path matching diagram is shown in Fig. 5. The optical path diagram in Fig. 5(a) shows that the light emitted by the SLD passes through the F-P scanning cavity and produces two transmitted light beams. The red arrow represents the transmitted light after the light emitted by the SLD directly passes through the F-P scanning cavity. Meanwhile, the blue arrow indicates the transmitted light generated after the light emitted by the SLD is transmitted for one circle in the F-P scanning cavity. After the two beams of light enter the sensor array, they are reflected by the reflective surfaces at the left and right ends of the sensor, as shown in Fig. 5(b). According to white light interferometry, when the optical paths of the two beams are matched, white light interference fringes are generated.

$$
\begin{gathered}
2 n L+2 n \sum_{i=1}^{j-1} l_{i}+2 n l_{j}=2 n L+2 n \sum_{i=1}^{j-1} l_{i}+2 X_{j} \\
j=1,2,3, \cdots, N
\end{gathered}
$$

where $n$ is the refractive index of the fiber core, $L$ is the fixed length of the fiber in the adjustable F-P cavity, $X$ is the adjustable distance of the scanning prism, and $X_{j}$ is the scanning distance from the $j$ th sensor.

(a)

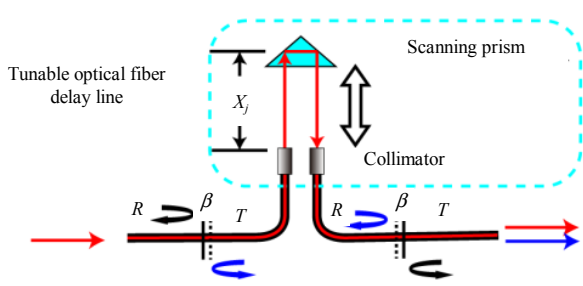

(b)

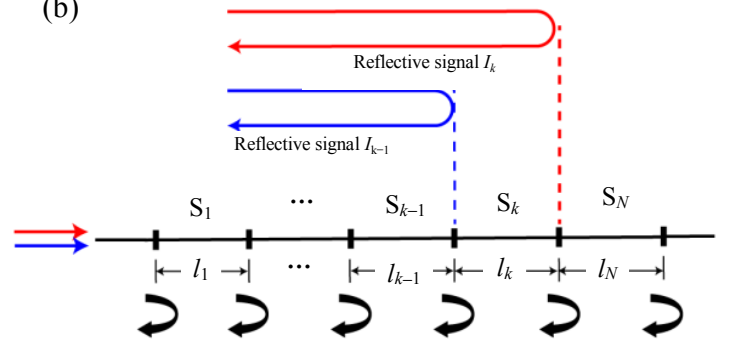

Fig. 5 Equivalent optical path diagram of the tunable fiber Fizeau cavity and sensor $k$ : (a) schematic diagram of the optical path in the F-P cavity and (b) optical path matching in the sensor array.

It can be seen from (1) that since the input signal and the reflected signal pass through the common optical path, this structure can realize automatic 
compensation for most temperature effects. If the adjustable F-P cavity is placed in the incubator, any fluctuation of the sensor optical path can be measured.

The variety of strain or ambient temperature of Sensor $j$ will change its optical path. Therefore, it is necessary to change the length of the adjustable F-P cavity to meet the optical path matching condition of (1). The relationship between the variety of adjustable distance and the variety of sensor length is as follows:

$$
\Delta X_{j}=\Delta\left(n l_{j}\right) \quad j=1,2,3, \cdots, N .
$$

For the sensor array, when the distributed stress is loaded on the sensor, we assume that the length of each sensor changes from $l_{1}$ to $l_{1}+\Delta l_{1}, l_{2}$ to $l_{2}+$ $\Delta l_{2}, \cdots$, and $l_{N}$ to $l_{N}+\Delta l_{N}$. The fiber delay line control system is used to precisely adjust the length of the F-P cavity to track the change of the sensor length. Because each sensor corresponds to a unique scanning prism position, the distributed strain can be calculated as follows:

$$
\varepsilon_{1}=\frac{\Delta l_{1}}{l_{1}}, \varepsilon_{2}=\frac{\Delta l_{2}}{l_{2}}, \cdots, \varepsilon_{N}=\frac{\Delta l_{N}}{l_{N}}
$$

where $\varepsilon_{j}$ is the strain value measured by the $j$ th sensor.

In order to avoid the measurement error caused by multiple reflections in the F-P cavity, the length of each optical fiber sensor needs to meet the requirements:

$$
\left\{\begin{array}{l}
l_{i} \neq l_{j} \\
n\left|l_{i}-l_{j}\right|_{\max }<D \\
n\left|l_{i}-l_{j}\right|_{\min }>\varepsilon_{\max }(k) l_{k}
\end{array} \quad i, j=1,2, \cdots, N\right.
$$

where $D$ is the maximum scanning distance of the stepper motor, and $\varepsilon_{\max }(k)$ is the maximum strain of all sensors.

To estimate the maximum multiplexing capability of the sensor based on the tunable F-P cavity fiber sensing system, we assume that the optical power of the injected fiber is $P_{0}$ and the minimum detection power of the photodetector is
$P_{\min }$. Then, the maximum number of sensors in the network can be estimated by the following formula:

$$
P_{D}(j) \geq P_{\min } \quad j=1,2, \cdots, N
$$

where $P_{D}(j)$ is the light intensity returned by the $j$ th sensor.

For an arbitrarily fiber sensor in the multiplex sensor array, the amplitude of the signal intensity output by the PD is proportional to the coherent term of the reflected signal at the two ends of the sensor.

$$
\begin{gathered}
P_{D}(j)= \\
\frac{1}{2} P_{0} T^{4} \beta^{4} \eta^{2}\left(X_{j}\right) R \sqrt{R_{j} R_{j+1}} T_{j} \beta_{j}\left[\prod_{i=1}^{j-1}\left(T_{i} \beta_{i}\right)\right]^{2}
\end{gathered}
$$

where $\beta$ is the insertion loss of connection between the optical fiber and tunable F-P cavity, and $T$ and $R$ represent the transmission coefficient and reflection coefficient of end face of the F-P cavity, respectively. $\beta_{j}$ represents the insertion loss of the $j$ th reflection end face of the sensor, and $T_{j}$ and $R_{j}$ represent the transmission coefficient and reflection coefficient of the $j$ th reflection end face, respectively. Due to the existence of $\beta_{j}$, the transmission coefficient $T_{j}$ is less than $1-R_{j} . \eta\left(X_{j}\right)$ is the insertion loss related to the tunable delay line of the optical fiber, which is a function of $X_{j}$.

We take the typical parameter $R=0.3, T=0.6$, $\beta=\beta_{j}=0.9(j=1,2, \cdots, N), R_{j}=1 \%$, and $T_{j}=$ 0.89 for theoretical simulation. Assume that the average loss of a tunable fiber delay line is $1.5 \mathrm{~dB}$, which means $\eta\left(X_{j}\right) \approx 0.7$, and the optical power injected into fiber is $P_{0}$. Generally, the typical detection capability of PDs in fiber sensing systems is about $1 \mathrm{nW}$. Considering the influence of noise background and other stray signals, the minimum detectable optical power of the detector is $P_{\min }=$ $5 \mathrm{nW}$. According to (6), when the output power of the SLD is $P_{0}=50 \mu \mathrm{W}$, the maximum number of sensors is $N_{\max }=4$; if $P_{0}=400 \mu \mathrm{W}$, the number of sensors increases to $N_{\max }=8$ [19].

For the space division multiplexing optical fiber white light interference sensing system, the estimation of the number of fiber sensors connected 
in the network is closely related to the optical path matching and dynamic scanning range of the spatial optical path. The maximum number of sensors is also limited by the scanning distance of the scanning prism. In the whole measurement range, if the lengths of the optical fiber sensor in the linear sensor array satisfy $l_{1}<l_{2}<\cdots<l_{N}$, the maximum number of sensors in the network is determined by the following formula:

$$
X_{v, \text { max }} / \max _{i=1,2, \cdots, N-1}\left\{l_{i+1}-l_{i}\right\}
$$

where $X_{v, \max }$ is the maximum scanning distance of the scanning prism and the maximum length difference between adjacent sensors. If $X_{v, \max }=$ $100 \mathrm{~mm}$ and $\max \left\{l_{i+1}-l_{i}\right\}=5 \mathrm{~mm}$, the maximum number of sensors is 20 .

In addition, the multiplexing scheme and topological structure of sensors in the network should be considered to estimate the noise of the sensing system. Then, the main noise should be suppressed in order to further improve the limit value $N_{\max }$ of the number of sensors in the network.

\section{Interrogation and applications of the white light interferometric sensor network}

Construction of an arbitrary OFSN is closely related to the specific system interrogation and sensor multiplexing methods. On the basis of the technologies discussed in the aforementioned general OFSN, this section reviews some specific interrogation and application schemes in the optical fiber white light interferometric sensor network.

\subsection{Applications of the linear OFSN based on white light interferometry}

Construction of a linear sensor array by optical fiber white light interferometry is the simplest and most commonly used network form in OFSNs. It is the most practical form of sensor multiplexing. And it is also the basis of white light interferometric OFSNs. Other types of network topologies are constructed by means of linear sensor arrays. In fact, there have been many such examples, and several typical white light interferometer demodulators can be used to construct this sensor array [20-24]. An example of a fiber-optic white light interferometric linear fiber sensor array based on the hetero-core fiber cascade structure, as shown in Fig. 6, will be further discussed.

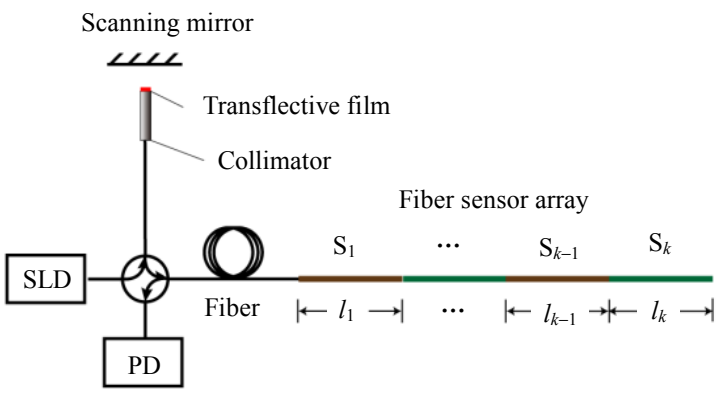

Fig. 6 Linear fiber sensor array based on the hetero-core fiber cascade structure.

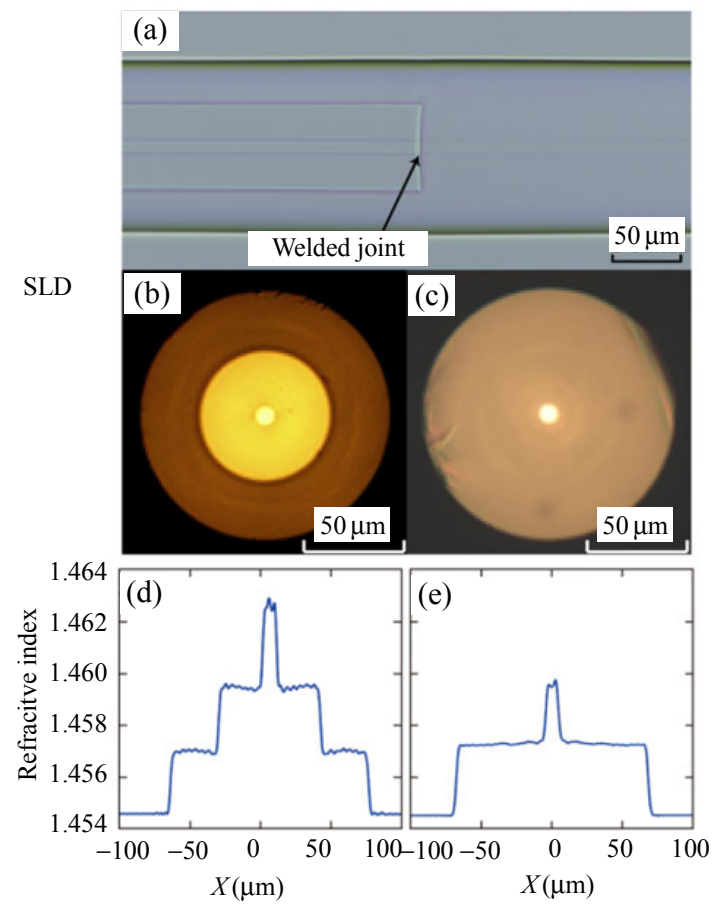

Fig. 7 Microscope image of the weak reflection structure formed at the fusion joint of DCF and SMF (a); microscope image and refractive index image of DCF (b, d), and SMF (c, e).

A quasi-distributed high temperature sensor array integrated in the fiber was proposed in [22]. The sensor array consists of a series of SMF and double-clad fiber (DCF) welded alternately. The details of the two kinds of optical fibers and their fusion joints are shown in Fig. 7. After the two optical fibers are fusion spliced, a weak reflection 
structure with extremely low reflectivity and low insertion loss is formed at the splice joint. Multiple weak reflection structures are cascaded to form an OFSN based on the white light interference technology. This method is a typical case of forming an OFSN through fusion splicing. Because the weak reflection structure has extremely low reflectivity and low insertion loss, more sensors can be multiplexed in the fiber sensor network. In addition, there are no other connectors in this OFSN. Therefore, it is small in size and can work at temperatures as high as $1000^{\circ} \mathrm{C}$ for a long time.

The distributed temperature sensing results of this sensor array in a high temperature environment are shown in the Fig. 8. The solid line in the figure is the test result of the thermocouple, and the scatter diagram is the test result of the distributed optical fiber sensor. It can be seen that the test results of the two are in good agreement.

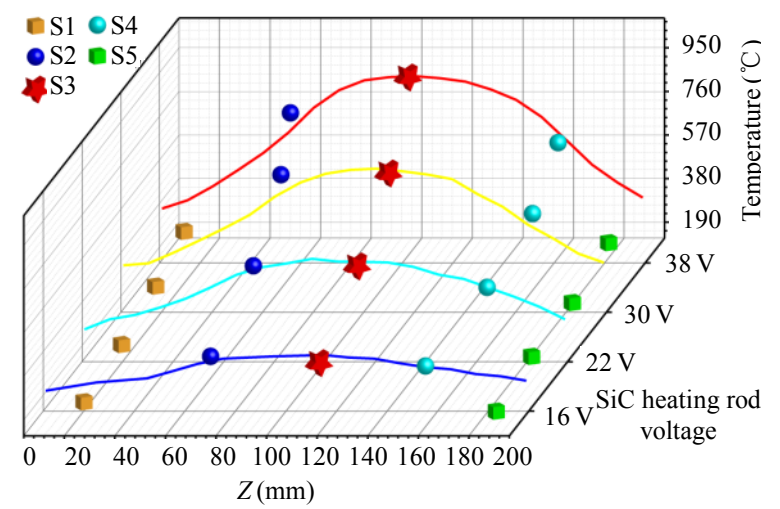

Fig. 8 Distributed temperature sensing results of the high temperature fiber sensor array.

\subsection{Star-type optical fiber sensor network technology}

Star-type OFSN is realized by a $1 \times N$ star-shaped optical fiber coupler, which realizes the parallel of the linear optical fiber sensor array and can form an optical fiber sensor matrix $[25,26]$.

Figure 9 shows a ring cavity FP interferometer with an adjustable cavity length. The tunable F-P cavity is used as an optical path inquiry device to query and demodulate the optical path change of each fiber sensor. The length of each optical fiber as a sensor can be selected to be slightly shorter than half the length of the F-P cavity. In this way, the optical signal delayed by the ring cavity can be compensated by the reflection of the two end faces of the optical fiber sensor. And the two optical signals reflected by the two end faces can reach the detector at the same time and cause interference. This kind of star-type OFSN requires a light source to have higher power. Because the optical power of the light source has to be distributed to each branch, the returned optical signal has to be attenuated by the $1 \times N$ star optical fiber coupler again. For an equally divided $1 \times N$ star fiber coupler, the signal of each fiber sensor is attenuated by a factor of $1 / N^{2}$. Therefore, the interrogation system uses a higher power amplified spontaneous emission (ASE) as the light source.

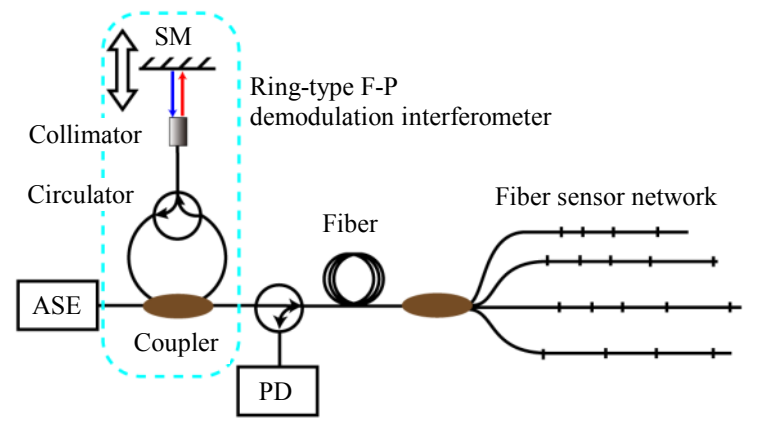

Fig. 9 Schematic diagram of star-type OFSN constructed based on an improved ring-type F-P optical path scanning interferometer (SM: scanning mirror).

\subsection{Ring-type optical fiber sensor network technology with dual port interrogation}

In order to realize dual-port inquiry, two new single-ring network multiplexed fiber optic strain sensing systems are shown in Fig. 10. Their structures are mainly composed of a $2 \times 2$ single-mode fiber coupler looped into a Sagnac fiber ring, and a number of sensors with slightly different lengths are distributed in the ring to distinguish positioning. The high-power ASE light source is connected to one port of the Sagnac fiber ring as the optical input, while the other port receives the reflected light signal from the fiber sensor and sends 
it to the interrogation interferometer to achieve optical path interrogation. This ring-type OFSN can have multiple interrogation methods. Figure 10 shows the structure of two typical white light interferometer demodulators. Figure 10(a) shows the asymmetrical Michelson interferometric optical path demodulator [27, 28], and Fig. 10(b) presents the unbalanced Mach-Zehnder (M-Z) interferometric optical path demodulator [29, 30].

(a)

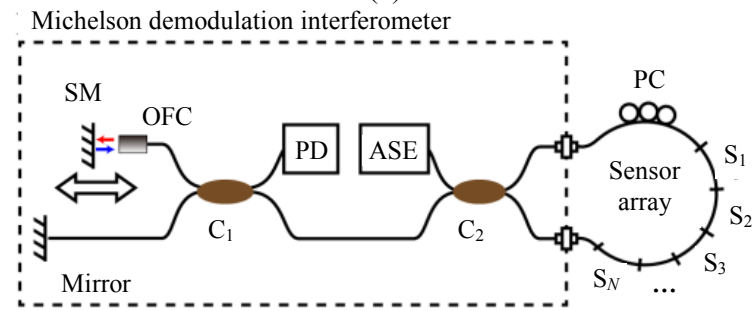

(b)

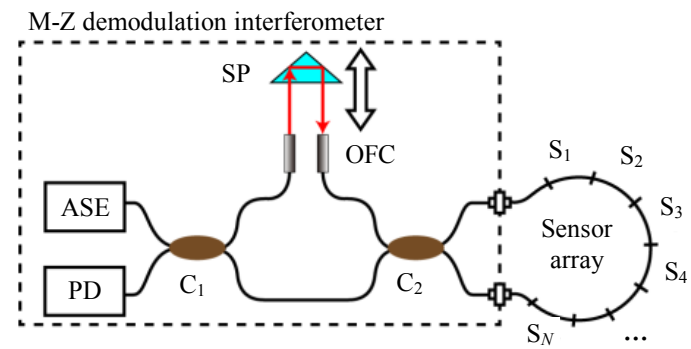

Fig. 10 Ring-type fiber sensor network based on Michelson (a) and Mach-Zehnder interferometer (b) [OFC: optical fiber collimator; SP: scanning prism; $\mathrm{C}_{1}, \mathrm{C}_{2}$ : couplers; $\mathrm{S}_{1}, \mathrm{~S}_{2}, \cdots, \mathrm{S}_{N}$ : sensors].

An ASE light source is used in the experiment. 10 sections of optical fibers are connected to each other and used as optical fiber sensors. The length of each sensor is about $1 \mathrm{~m}$. The length difference between sensors is about $7 \mathrm{~mm}$ and they are connected through ceramic ferrules. The strength distributions of the output signals of 10 sensor networks when the ring network is in the closed loop and open loop are shown in Fig. 11, respectively.

It can be seen from the experimental results that the height of each signal peak reflects the intensity of the coherent signal reflected from both ends of the sensor. The result is approximately consistent with the theoretical prediction, but has a large fluctuation. The reason is that it is difficult to ensure that the reflectivity of each fiber segment is exactly the same as the theoretical value in the experiment.

It can be seen that the results shown in Figs. 11(a) and 11(b) basically give the same measurement information about the peak position. This result shows that even if the loop of the fiber sensor network is broken, the sensor system can still work as usual. In addition, the closed-loop signal strength is higher than that in the open-loop state.

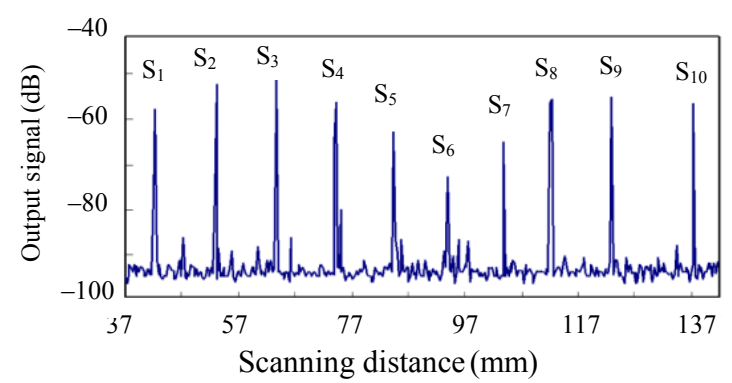

(a)

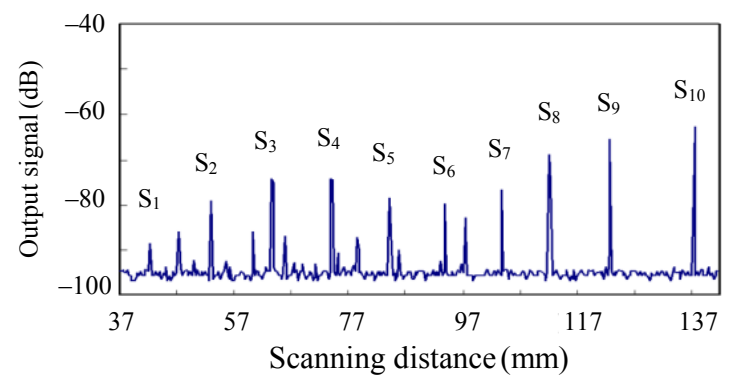

(b)

Fig. 11 Test results of the sensor network composed of 10 fiber optic sensors: (a) closed loop and (b) open loop.

Based on the results of the aforementioned single-ring network construction, the double-ring network structure of the white light interference sensor composed of two ring structures can be further constructed [31, 32]. The dual-ring topology of the fiber sensor network is completely passive, and the absolute length can be measured through each sensing fiber. Therefore, it can be used for strain and temperature quasi-distributed measurement. For large-scale smart structures, this technology not only can expand the potential for multiplexing, but also can provide sensors with redundancy against damage. The research results show that the coupled dual-loop sensor network 
allows two breakpoints. Even if the embedded dual-loop sensor is damaged somewhere, the sensor system can still work as usual. The robustness of the 9-sensor dual-loop sensor network was discussed and demonstrated in detail [32]. The coupled dual-ring topology network structure of the fiber sensor system not only can expand the multiplexing capacity of the sensor network, but also can provide redundancy to meet the requirements for the anti-damage and reliability of the sensor system. This method can effectively avoid the failure of the entire fiber sensor system when the embedded sensor link is broken.

Figure 12 shows a dual ring-type sensor network constructed by interconnecting two ring topology structures. The research results show that the ring-type fiber sensor network multiplexing technology has some outstanding advantages [32]. (1) Comparing the linear sensor network and star sensor network structure, the ring structure increases the signal output amplitude of the sensor. Especially compared with the sensors at the end of the serial line array network, this kind of amplification effect is particularly obvious. (2) The multiplexing ability of the sensor network is improved. Under the premise of the same input optical power, more sensors can be connected. (3) The anti-damage ability of the OFSN is enhanced. Even if individual sensors in the network fail, the ring structure divides into two linear sensor networks. Due to the dual-port interrogation technology, the overall failure of the sensor network system is effectively avoided.

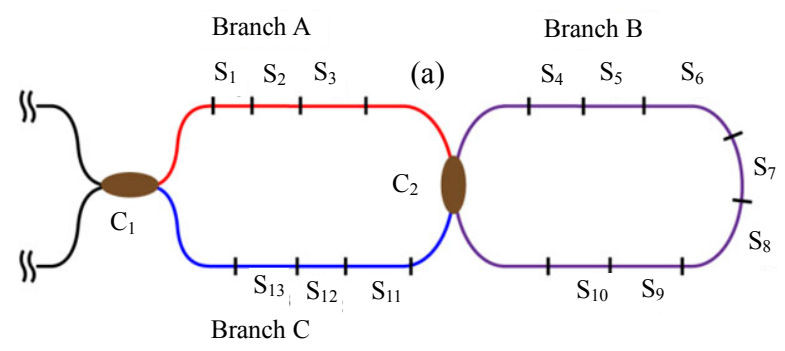

Fig. 12 Dual ring-type fiber sensor network constructed by interconnecting two ring topology structures.

\subsection{Bus-type optical fiber sensor network technology}

The advantage of the bus-type white light interferometric OFSN is that multiple linear fiber sensor arrays can be arbitrarily connected through a series of $1 \times 2$ optical fiber couplers. Each linear sensor branch is independent of each other, so that a failure of the arbitrary sensor network branch will not affect other branches [33].

Figure 13 shows a bus-type optical fiber white light interferometric sensor network structure constructed by the Fizeau scanning interferometer. In this network topology, $1 \times 2$ fiber coupler is the main optical splitting device in the sensor system, which is responsible for distributing the optical power to each branch. In order to make the signal of each sensor in the system finally reach the photodetector relatively balanced, the split ratio of each coupler $\mathrm{C}_{j}$ needs to be optimized.

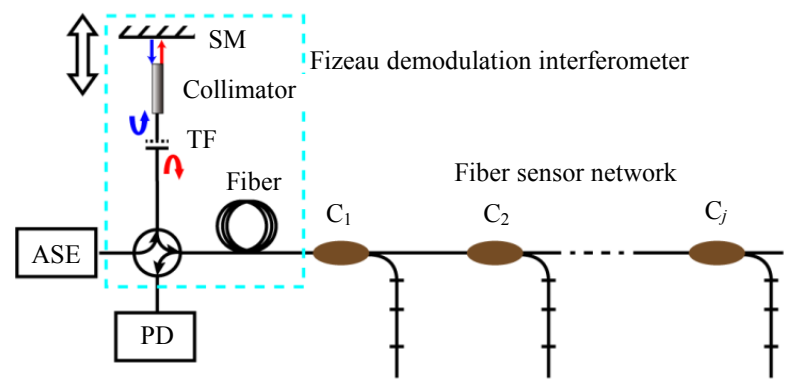

Fig. 13 Schematic diagram of bus-type optical fiber white light interference sensor network constructed by Fizeau scanning interferometer $\left(\mathrm{C}_{1}, \mathrm{C}_{2}, \cdots, \mathrm{C}_{j}\right.$ : couplers; TF: transflective film).

Assume that the splitting ratio $S R_{j}$ of the $j$ th fiber coupler is

$$
S R_{j}=\frac{\tau_{j}}{\kappa_{j}}=\frac{\tau_{j}}{1-\tau_{j}}
$$

where $\kappa_{j}$ and $\tau_{j}$ represent transmitted energies of two arms of the $j$ th coupler, respectively.

In order to make the optical power of the sensor signal of each branch detected by the photodetector approximately equal, we use the signal power that is reflected back to the photodetector by the first sensor of each branch as a reference for calculation, as shown in Fig. 14. The optical power received by 
the detector $P_{j}$ can be calculated as

$$
P_{j}=I_{0} \xi R_{j} \prod_{i=1}^{N} \tau_{i}\left(1-\tau_{i}\right)
$$

where $I_{0}$ is the optical power of ASE, $\xi$ represents the total attenuation coefficient of various optical powers of the interrogation system, and $R_{j}$ is the reflection coefficient of the $j$ th sensor. Through (8) and (9), the split ratio parameters of each fiber coupler in the bus-type fiber optic sensor network can be optimized according to the number of branches of the branch linear network.

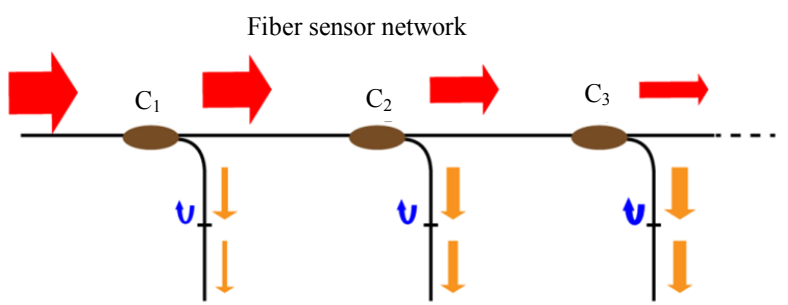

Fig. 14 Schematic diagram of power distribution of each branch in the bus-type OFSN.

\subsection{Ladder-type optical fiber sensor network technology}

The ladder-type white light interferometric strain sensor network is shown in Fig. 15. This network topology can be regarded as a combination of a ring network and a bus network. Therefore, this network structure has the advantages of both structures. On the one hand, its bus structure makes each ladder branch independent, and it will not affect normal operation of the entire system due to the damage of a certain ladder branch. On the other hand, each ladder branch and the entire system form a dual-port query ring-type sensor network. Even if a breakpoint occurs somewhere in the ladder branch, the information of each sensor can be obtained through both ends [34].

This section discusses how to construct a sensor network based on the optical fiber white light interference technology. In addition, taking a simple structure of the optical fiber strain sensor as an example, the construction of the optical fiber white light interference strain sensor network is discussed.
The reason why fiber optic sensor networks are becoming more and more attractive is mainly due to the following two aspects. The first point is that sensor networks are required to have long-distance testing capabilities, and they are usually required to have high multiplexed capability. The second point is the need to reduce the price of a single sensor in the fiber sensor network. But so far, only quite a few systems can realize the construction of large-scale sensor networks. For optical fiber white light interferometric sensors, most optical fiber sensor network systems contain only a few sensors. In order to break this limitation, a lot of work shall be done in the future, such as how to reduce the internal noise of the optical fiber sensor network system and improve the optical path scanning range of the white light interference system $[35,36]$.

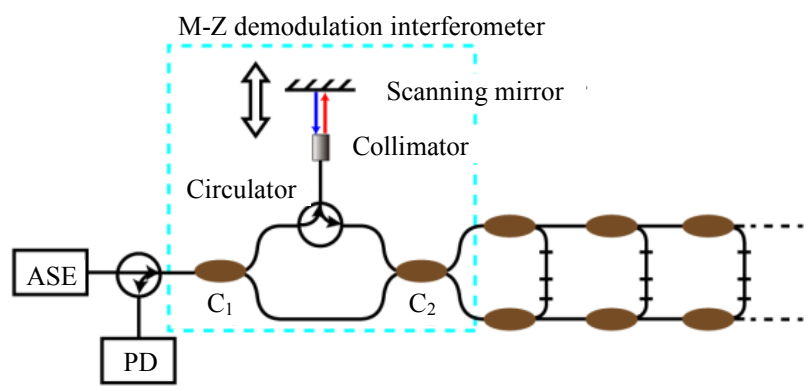

Fig. 15 Schematic diagram of ladder-type fiber white light interferometric sensing network constructed by the Mach-Zehnder scanning interferometer.

\section{Conclusions}

The main characteristics of the OFSN system include: network topology, multiplexed capability of sensors, the length of the guiding fiber and its insensitivity, sensor sensitivity, and dynamic range, power consumption estimation (including light source power, noise source, loss, and detector sensitivity), reliability, and cost. Optical fiber has the ability to simultaneously sense signals and transmit signals. This ability will become a powerful driving force for the development of optical fiber sensors. However, only a few systems can realize the construction of large-scale sensor networks. For 
optical fiber sensors based on the white light interference technology, most optical fiber sensor network systems only contain a small number of sensors. How to further reduce the loss of constructing sensors and improve the efficiency of light source utilization is the development direction of the OFSN technology based on the white light interference technology. It can be expected that for a long period of time in the future, the OFSN will be an important field of optical fiber sensing and it will be widely used in future engineering systems.

Open Access This article is distributed under the terms of the Creative Commons Attribution 4.0 International License (http://creativecommons.org/licenses/by/4.0/), which permits unrestricted use, distribution, and reproduction in any medium, provided you give appropriate credit to the original author(s) and the source, provide a link to the Creative Commons license, and indicate if changes were made.

\section{References}

[1] Q. Chai, Y. Luo, J. Ren, J. Zhang, J. Yang, L. Yuan, et al., "Review on fiber-optic sensing in health monitoring of power grids," Optical Engineering, 2019, 58(7): 072007.

[2] P. Xu, D. Ba, W. He, H. Hu, and Y. Dong, "Distributed Brillouin optical fiber temperature and strain sensing at a high temperature up to $1000^{\circ} \mathrm{C}$ by using an annealed gold-coated fiber," Optics Express, 2018, 26(23): 29724-29734.

[3] D. Liu, Q. Sun, P. Lu, L. Xia, and C. Sima, "Research progress in the key device and technology for fiber optic sensor network," Photonic Sensors, 2016, 6(1): 1-25.

[4] X. He, Z. Ran, T. Yang, Y. Xiao, Y. Wang, and Y. Rao, "Temperature-insensitive fiber-optic tip sensors array based on OCMR for multipoint refractive index measurement," Optics Express, 2019, 27(7): 9665-9675.

[5] Y. Wang, J. Gong, D. Y. Wang, B. Dong, W. Bi, and A. Wang, "A quasi-distributed sensing network with time-division-multiplexed fiber Bragg gratings," IEEE Photonics Technology Letters, 2011, 23(2): 70-72.

[6] Z. Wang, F. Shen, L. Song, X. Wang, and A. Wang, "Multiplexed fiber Fabry-Pérot interferometer sensors based on ultrashort Bragg gratings," IEEE Photonics Technology Letters, 2007, 19(8): 622-624.

[7] K. Stępień, M. Slowikowski, T. Tenderenda, M. Murawski, M. Szymanski, L. Szostkiewicz, et al.,
"Fiber Bragg gratings in hole-assisted multicore fiber for space division multiplexing," Optics Letters, 2014, 39(12): 3571-3574.

[8] J. Huang, X. Lan, M. Luo, and H. Xiao, "Spatially continuous distributed fiber optic sensing using optical carrier based microwave interferometry," Optics Express, 2014, 22(15): 18757-18769.

[9] T. G. Liu, Z. Yu, J. F. Jiang, K. Liu, X. Z. Zhang, Z. Y. Ding, et al., "Advances of some critical technologies in discrete and distributed optical fiber sensing research," Acta Physica Sinica, 2017, 66(7): 070705.

[10] B. A. Childers, M. E. Froggatt, S. G. Allison, T. C. Moore, D. A. Hare, C. F. Batten, et al., "Use of 3000 Bragg grating strain sensors distributed on four 8-m optical fibers during static load tests of a composite structure," SPIE, 2001, 4332: 133-142.

[11] M. Zhang, Q. Sun, Z. Wang, X. Li, H. Liu, and D. Liu, "A large capacity sensing network with identical weak fiber Bragg gratings multiplexing," Optics Communications, 2012, 285(13-14): 3082-3087.

[12] Y. J. Rao, A. B. Lobo Ribeiro, D. A. Jackson, L. Zhang, and I. Bennion, "Simultaneous spatial, time and wavelength division multiplexed in-fibre grating sensing network," Optics Communications, 1996, 125(1-3): 53-58.

[13] M. Yang, W. Bai, H. Guo, H. Wen, H. Yu, and D. Jiang, "Huge capacity fiber-optic sensing network based on ultra-weak draw tower gratings," Photonic Sensors, 2016, 6(1): 26-41.

[14] E. Zhao, Y. Yuan, J. Yang, A. Zhou, and L. Yuan, “A novel multiplexed fiber optic deformation sensing scheme," Sensor Letters, 2012, 10(7): 1526-1528.

[15] A. Yan, S. Li, Z. Peng, R. Zou, P. Ohodnicki, P. Lu, et al., "Multi-point fiber optic sensors for real-time monitoring of the temperature distribution on transformer cores," SPIE, 2018, 10639: 1063912.

[16] Z. Qu, S. Guo, C. Hou, J. Yang, and L. Yuan, "Real-time self-calibration PGC-Arctan demodulation algorithm in fiber-optic interferometric sensors," Optics Express, 2019, 27(16): 23593-23609.

[17] S. Li, G. Lu, C. Lai, Y. Huang, and Y. En, "Optical-path difference on-line measurement of multiplexing fiber-optic interferometric sensors using TDM and WDM by improved opticalfrequency-domain reflectometry," SPIE, 2019, 11340: $113400 \mathrm{~N}$.

[18] M. Wang, Y. Yang, S. Huang, J. Wu, K. Zhao, Y. Li, et al., "Multiplexable high-temperature stable and low-loss intrinsic Fabry-Perot in-fiber sensors through nanograting engineering," Optics Express, 2020, 28(14): 20225-20235.

[19] L. Yuan and Y. Dong, "Multiplexed fiber optic twin-sensor array based on a combination of Mach-Zehnder and Michelson interferometers," Journal of Intelligent Material Systems and 
Structures, 2009, 20(7): 809-813.

[20] Y. Yuan, B. Wu, J. Yang, and L. Yuan, "Tunable optical-path correlator for distributed strain or temperature-sensing application," Optics Letters, 2010, 35(20): 3357-3359.

[21] W. Li, Y. Yuan, J. Yang, H. Deng, and L. Yuan, "In-fiber integrated sensor array with embedded weakly reflective joint surface," Journal of Lightwave Technology, 2018, 36(23): 5663-5668.

[22] W. Li, Y. Yuan, J. Yang, and L. Yuan, "In-fiber integrated quasi-distributed high temperature sensor array," Optics Express, 2018, 26(26): 3411334121 .

[23] Y. Zhao, S. Huang, Z. Cui, Q. Chai, Y. Liu, J. Ren, et al., "Electric-arc-induced strength-controllable weak polarization mode coupling in polarization maintaining fiber," Applied Optics, 2018, 57(22): 6446.

[24] W. Li, Y. Yuan, J. Yang, and L. Yuan, "In-fiber integrated high sensitivity temperature sensor based on long Fabry-Perot resonator," Optics Express, 2019, 27(10): 14675-14683.

[25] L. Yuan and L. Zhou, " $1 \times N$ star coupler as a distributed fiber-optic strain sensor in a white-light interferometer," Applied Optics, 1998, 37(19): 4168-4172.

[26] Y. J. Rao and D. A. Jackson, "Prototype multiplexing system for use with a large number of fiber optic based extrinsic Fabry-Perot sensors exploiting low-coherence interrogation," SPIE, 1995, 2507: 90-98.

[27] L. Yuan, W. Jin, L. Zhou, Y. L. Hoo, and M. S. Demokan, "Enhanced multiplexing capacity of low-coherence reflectometric sensors with a loop topology," IEEE Photonics Technology Letters, 2002, 14(8): 1157-1159.

[28] L. Yuan, W. Jin, L. Zhou, Y. L. Hoo, and M. S. Demokan, "Enhancement of multiplexing capability of low-coherence interferometric fiber sensor array by use of a loop topology," Journal of Lightwave Technology, 2003, 21(5): 1313-1319.

[29] L. Yuan, L. Zhou, W. Jin, and J. Yang, "Low-coherence fiber-optic sensor ring network based on a Mach-Zehnder interrogator," Optics Letters, 2002, 27(11): 894-896.

[30] L. Yuan, L. Zhou, W. Jin, and J. Yang, "Design of a fiber-optic quasi-distributed strain sensors ring network based on a white-light interferometric multiplexing technique," Applied Optics, 2002, 41(34): 7205-7211.

[31] L. Yuan and J. Yang, “Two-loop-based low-coherence multiplexing fiber-optic sensor network with a Michelson optical path demodulator," Optics Letters, 2005, 30(6): 601-603.

[32] J. Yang, L. Yuan, and W. Jin, "Improving the reliability of multiplexed fiber optic low-coherence interferometric sensors by use of novel twin-loop network topologies," Review of Scientific Instruments, 2007, 78(5): 055106.

[33] S. Li, F. Mokhtar, and L. Yuan, "Multi-array sensors tree network based on white light fiber-optic Mach-Zehnder interferometer," Sensor Letters, 2012, 10(7): 1378-1381.

[34] S. Li, L. Yuan, and F. Mokhtar, "Ladder topology network based on white light fiber-optic Mach-Zehnder interferometer," SPIE, 2011, 8199: 819918.

[35] Y. Yuan, D. Lu, J. Yang, J. Wang, H. Li, Z. Yu, et al., "Range extension of the optical delay line in white light interferometry," Applied Optics, 2017, 56(16): 4598-4605.

[36] Y. Yuan, Y. Cheng, J. Yang, H. Zhang, D. Lu, Y. Lv, et al., "Suppression of interference noise caused by Fresnel reflection in all-fiber white-light interferometer," Applied Optics, 2017, 56(31): 8732-8737. 